\title{
Keissleriella dactylidis, sp. nov., from Dactylis glomerata and its phylogenetic placement
}

\author{
Chonticha Singtripop ${ }^{\mathrm{a}, \mathrm{b}, \mathrm{c}}$, Erio Camporesi ${ }^{\mathrm{d}, \mathrm{e}, \mathrm{f}}$, Hiran A. Ariyawansa ${ }^{\mathrm{c}}$, Dhanushka N. Wanasinghe ${ }^{\mathrm{c}}$, \\ Ali H. Bahkall ${ }^{\text {, Putarak Chomnunti }}{ }^{\mathrm{c}}$, Saranyaphat Boonmee ${ }^{\mathrm{c}}$, Kevin D. Hyde , $^{\mathrm{a}, \mathrm{b}, \mathrm{g}, *}$ \\ ${ }^{a}$ Key Laboratory of Biodiversity and Biogeography, Kunming Institute of Botany, \\ Chinese Academy of Sciences, Kunming 650201, China \\ b World Agroforestry Centre, East and Central Asia, 132 Lanhei Road, Kunming 650201, China \\ c Institute of Excellence in Fungal Research, Mae Fah Luang University, Chiang Rai 57100 Thailand \\ d A.M.B. Gruppo Micologico Forlivese "Antonio Cicognani", Via Roma 18, Forlì, Italy \\ e A.M.B. Circolo Micologico "Giovanni Carini", C.P. 314, Brescia, Italy \\ f Società Per Gli Studi Naturalistici della Romagna, C.P. 144, Bagnacavallo (RA), Italy \\ g College of Science, Botany and Microbiology Department, King Saud University, Riyadh, KSA, 1145, \\ Saudi Arabia
}

*Corresponding author, e-mail: kdhyde3@gmail.com

Received 26 May 2015

Accepted 1 Nov 2015

\begin{abstract}
A new species of Keissleriella (Lentitheciaceae) is described from a dead stem of Dactylis glomerata collected in Italy. The new taxon is supported by morphology and phylogenetic analysis of SSU, LSU, and TEF1 $\alpha$ sequence data. The combined analysis of gene data indicate that Keissleriella dactylidis sp. nov. forms a distinct clade within the genus Keissleriella. The species is characterized by immersed, erumpent, dark grey to black ascomata with papilla containing brown periphyses, and cylindrical asci with hyaline, ellipsoid, 4-5-septate ascospores with a thin mucilaginous sheath. A synopsis of the characteristics of Keissleriella dactylidis and other species is provided and the new species is justified and compared with similar taxa. This is the first report of a Keissleriella species from the grass Dactylis glomerata.
\end{abstract}

KEYWORDS: Dactylidis, Gramineae, Keissleriella, Lentitheciaceae, Poaceae

\section{INTRODUCTION}

Dothideomycetes is the largest and most diverse class of Ascomycota comprising an estimated 19000 species $^{1,2}$ and is characterized by bitunicate, usually fissitunicate $\operatorname{asci}^{1,3}$. The majority of species in this class are endophytes, epiphytes, or saprobes, with a small number occurring as lichens and hyperparasites ${ }^{1,4}$. Many of the asexual morphs of dothideomycetous are pathogens of economically important plants and crops $s^{5,6}$.

Pleosporales is the largest order in Dothideomycetes, comprising a quarter of all dothideomycetous species ${ }^{7,8}$ and comprising 41 families $^{1}$. This large order comprises two main suborders, namely Pleosporineae and Massarineae. Pleosporineae includes nine families: Cucurbitariaceae, Didymellaceae, Didymosphaeriaceae, Dothidotthiaceae, Leptosphaeriaceae, Phaeosphaeriaceae, Pleosporaceae $^{9}$, Halojulellaceae, and Shiraiaceae ${ }^{10,11}$, while Massarineae includes six families: Bambusicola- ceae $^{1}$, Montagnulaceae, Lentitheciaceae, Massarinaceae, Morosphaeriaceae, and Trematosphaeriaceae $^{2}$. Ariyawansa et $\mathrm{al}^{7}$ have however synonymized Montagnulaceae under Didymosphaeriaceae by giving the priority to the oldest name.

The family Lentitheciaceae was introduced by Zhang et $\mathrm{al}^{12}$ in the order Pleosporales. Most members in this family are saprobic on stems and twigs of herbaceous and woody plants in terrestrial or aquatic habitats ${ }^{12,13}$. The family is characterized by the lenticular ascomata, ostioles with or without brown setae, and hyaline, sometimes light yellow and 1-septate to multi-septate ascospores ${ }^{1,9}$.

Keissleriella was introduced by Höhnel ${ }^{14}$ and is typified by Keissleriella aesculi Sacc. (三 Pyrenochaeta aesculi Höhn.). The genus Keissleriella is characterized by ascomata with papilla filled with black setae, and hyaline and 1 to multi-septate ascospores ${ }^{15-17}$. Previous studies based on morphology and multigene analysis have shown that the natural classification of Keissleriella is confusing, thus several authors 
classified the genus under different families. For example, Munk ${ }^{18}$ placed this genus in Lophiostomataceae (including Massarinaceae), Arx and Müller ${ }^{19}$ placed it in Pleosporaceae, and Barr ${ }^{15}$ referred it to Melanommataceae. Lumbsch and Huhndorf ${ }^{20}$ classified Keissleriella in the family Massarinaceae and Zhang et al ${ }^{12}$ placed the genus in Lentitheciaceae.

The aim of this paper is to introduce and describe a new Keissleriella species which was discovered as a result of examining saprobic fungi on Dactylis glomerata in Italy and discuss its taxonomic placement in Lentitheciaceae based on morphology and phylogeny.

\section{MATERIAL AND METHODS}

\section{Sample collection and morphological studies}

Dead stems of Dactylis glomerata were collected on 5 August 2012 in Marilleva in the Province of Trento, Italy and brought to the laboratory in Ziplock plastic bags where they were examined under a Motic SMZ 168 series dissecting stereo-microscope. Hand sections of ascomata were mounted in 10\% lactoglycerol for microscopic studies and photomicrography. The fungus was examined using a Nikon ECLIPSE 80i compound microscope and photographed by a Canon 450D digital camera fitted to the microscope. Measurements were made by TAROSOFT IMAGE FRAME WORK and images used for figures were made with Adobe PHotoshop CS3 EXTENDED 10.0 (Adobe Systems Inc., US). Isolation was carried out from single ascospores following the method described by Chomnunti et $\mathrm{al}^{21}$ and germinating single spores were transferred directly to malt extract agar (MEA) plates and grown at $15-18^{\circ} \mathrm{C}$. Characters of colonies were observed and measured after 12 weeks.

Herbarium specimens were obtained on loan from the Research Institute of Resource Insects (RIRI), Kunming, China.

The type material is deposited in the herbarium of Mae Fah Luang University (MFLU), Chiang Rai, Thailand and New Zealand Fungal and Plant Disease Collection (PDD), New Zealand. Cultures are deposited at the Mae Fah Luang University Culture Collection (MFLUCC) and Centraalbureau Voor Schimmelcultures (CBS).

\section{DNA extraction and PCR reaction}

Genomic DNA was extracted from mycelium grown on MEA for $1-2$ weeks at $15-18^{\circ} \mathrm{C}$ using a Biospin Fungus Genomic DNA Extraction Kit (BioFlux) following the manufacturer's protocol. The amplifi- cation of rDNA regions of the small subunit rDNA (SSU), large subunit (LSU) and translation elongation factor 1-alpha gene (TEF1 $\alpha$ ) was carried out by using NS1 and NS4 ${ }^{22}$, LROR and LR5 ${ }^{23}$, and EF1983F and EF1-2218R ${ }^{24}$ primer pairs, respectively. The amplification procedure was performed in a $25 \mu \mathrm{l}$ reaction volume containing $1 \mu \mathrm{l}$ DNA, $1 \mu \mathrm{l}$ of primer 1 and primer $2,12.5 \mu \mathrm{l}$ of $2 \times$ Master Mix and $9.5 \mu \mathrm{l}$ of $\mathrm{ddH}_{2} \mathrm{O}$. Amplification conditions were setup for initial denaturation of $3 \mathrm{~min}$ at $94^{\circ} \mathrm{C}$, followed by 35 cycles of $30 \mathrm{~s}$ at $94^{\circ} \mathrm{C}, 50 \mathrm{~s}$ at $55^{\circ} \mathrm{C}$ and $1 \mathrm{~min}$ at $72^{\circ} \mathrm{C}$, and a final extension period of 10 min at $72^{\circ} \mathrm{C}$. The PCR products were checked on $1 \%$ agarose electrophoresis gels stained with ethidium bromide. The purification and sequencing of PCR products were sent to Shanghai Majorbio Biopharm Technology Co., Ltd, China.

\section{Sequence alignment and phylogenetic analysis}

The newly generated sequences were compared with other sequences in GenBank by nucleotide megablast searches. Multiple sequence alignment was carried out in BIOEDIT ${ }^{25}$ and Clustal $\mathrm{X}^{26}$. Maximum-parsimony (MP) analysis was processed using PAUP v. $4.0 \mathrm{~b} 10^{27}$ to obtain the most parsimonious tree. Clade stability was assessed in a bootstrap analysis with 1000 replicates, random sequence additions with max-trees set to 1000 and other default parameters as implemented in PAUP. The setup detail of the above phylogenetic analysis programs were followed as described by Kishino and Hasegawa ${ }^{28}$. Maximum trees were viewed in Treeview $^{29}$.

\section{RESULTS AND DISCUSSION \\ DNA sequencing-SSU, LSU, and TEF rDNA phylogenies}

The combined gene analysis of the SSU, LSU, and TEF dataset comprised 55 taxa including our new stain of Keissleriella dactylidis (MFLUCC 13-0751) with Halojulella avicenniae as the outgroup taxon (BCC 18422). This analysis comprised 2517 characters, of which 1767 were constant, 542 variable characters were parsimony informative and 208 variable characters are parsimony-uninformative. Six equally parsimonious trees were generated and the first of the most parsimonious tree was selected (Fig. 1). Bootstrap support values of MP (equal to or above $50 \%$ based on 1000 replicates) are shown on the upper branches. The Kishino-Hasegawa test shows length $=2161$ steps with $\mathrm{CI}=0.460, \mathrm{RI}=$ $0.645, \mathrm{RC}=0.296$, and $\mathrm{HI}=0.540$. 


\section{Phylogeny}

Our strain Keissleriella dactylidis (MFLUCC 13-0751) groups with strains of Keissleriella in the fam- ily Lentitheciaceae, but are separated from other species in the genus with relatively high bootstrap support (65\%, Fig. 1) and form a robust clade sister to Keissleriella linearis.

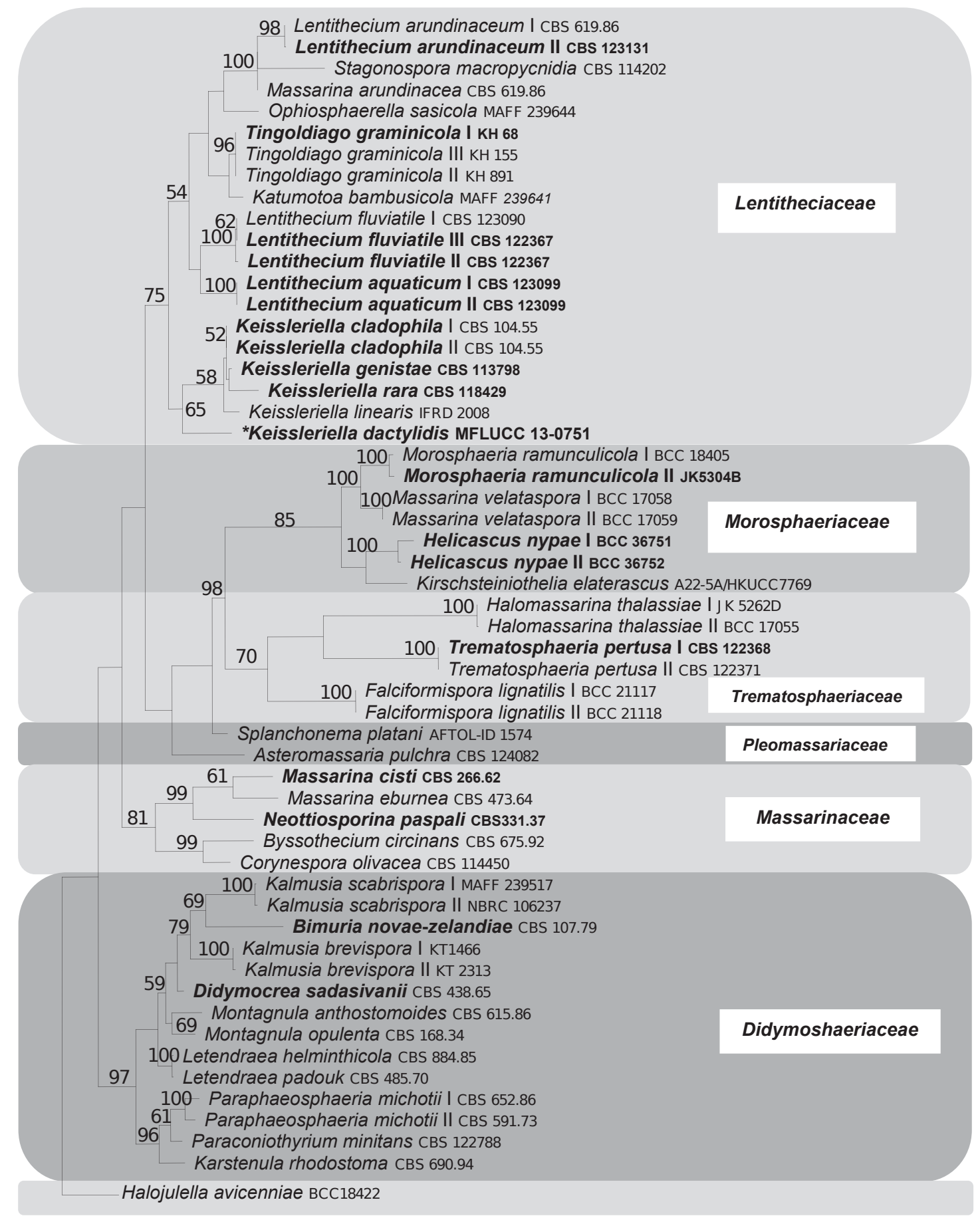

Fig. 1 Gene tree constructed using MP method based on a combined dataset of SSU, LSU, and TEF genes sequence data. Halojulella avicenniae is the outgroup taxon. Bootstrap support values $>50 \%$ are shown above the branch. All type strains are in bold and the original isolate numbers are noted after the species names. 
Table 1 Taxa used in the phylogenetic analysis and their corresponding GenBank Accession numbers.

\begin{tabular}{|c|c|c|c|c|}
\hline \multirow[t]{2}{*}{ Species } & \multirow[t]{2}{*}{ Culture Accession No. } & \multicolumn{3}{|c|}{ GenBank Accession No. } \\
\hline & & LSU & SSU & TEF1 \\
\hline Asteromassaria pulchra ${ }^{\dagger}$ & CBS 124082 & GU301800 & GU296137 & GU349066 \\
\hline Bimuria novae zelandiae & CBS 107.79 & AY016356 & AY016338 & DQ471087 \\
\hline Byssothecium circinans ${ }^{\dagger}$ & CBS 675.92 & AY016357 & AY016339 & GU349061 \\
\hline Corynespora olivacea ${ }^{\dagger}$ & CBS 114450 & GU301809 & - & GU349014 \\
\hline Didymocrea sadasivanii & CBS 438.65 & DQ384103 & DQ384066 & - \\
\hline Falciformispora lignatilis $^{\dagger}$ & BCC 21117 & GU371826 & GU371834 & GU371819 \\
\hline Falciformispora lignatilis $^{\dagger}$ & BCC 21118 & GU371827 & GU371835 & GU371820 \\
\hline H. avicenniae $\uparrow$ & BCC 18422 & GU371823 & GU371831 & GU371816 \\
\hline Halomassarina thalassiae ${ }^{\dagger}$ & JK 5262D & GU301816 & - & GU349011 \\
\hline Halomassarina thalassiae ${ }^{\dagger}$ & BCC 17055 & GQ925850 & GQ925843 & - \\
\hline Helicascus nypae & BCC 36751 & GU479788 & GU479754 & GU479854 \\
\hline Helicascus nypae & BCC 36752 & GU479789 & GU479755 & GU479855 \\
\hline Kalmusia brevispora & KT1466 & AB524600 & AB524459 & AB539112 \\
\hline Kalmusia brevispora ${ }^{\dagger}$ & KT 2313 & AB524601 & AB524460 & AB539113 \\
\hline Kalmusia scabrispora ${ }^{\dagger}$ & MAFF 239517 & AB524593 & AB524452 & AB539106 \\
\hline Kalmusia scabrispora ${ }^{\dagger}$ & NBRC 106237 & AB524594 & AB524453 & AB539107 \\
\hline Karstenula rhodostoma ${ }^{\dagger}$ & CBS 690.94 & GU301821 & GU296154 & GU349067 \\
\hline Katumotoa bambusicola $^{\dagger}$ & MAFF 239641 & AB524595 & AB524454 & AB539108 \\
\hline Keissleriella cladophila & CBS 104.55 & GU205221 & GU205241 & - \\
\hline Keissleriella cladophila & CBS 104.55 & GU301822 & GU296155 & GU349043 \\
\hline Keissleriella dactylidis & MFUCC13-0751 & KP197668 & KP197666 & KP197669 \\
\hline Keissleriella genistae & CBS 113798 & GU205222 & GU205242 & - \\
\hline Keissleriella rara & CBS 118429 & GU479791 & GU479757 & - \\
\hline Keissleriella linearis ${ }^{\dagger}$ & IFRD 2008 & FJ795435 & & \\
\hline Kirschsteiniothelia elaterascus ${ }^{\dagger}$ & A22-5A/HKUCC7769 & AY787934 & AF053727 & - \\
\hline Lentithecium aquaticum & CBS 123099 & FJ795434 & FJ795477 & - \\
\hline Lentithecium aquaticum & CBS 123099 & GU301823 & GU296156 & GU349068 \\
\hline Lentithecium arundinaceum ${ }^{\dagger}$ & CBS 619.86 & GU301824 & GU296157 & - \\
\hline Lentithecium arundinaceum & CBS 123131 & GU456320 & GU456298 & GU456281 \\
\hline Lentithecium fluviatile $\left.\right|^{\dagger}$ & CBS 123090 & FJ795450 & FJ795492 & - \\
\hline Lentithecium fluviatile $^{\dagger}$ & CBS 122367 & FJ795451 & FJ795493 & - \\
\hline Lentithecium fluviatile $^{\dagger}$ & CBS 122367 & GU301825 & GU296158 & GU349074 \\
\hline Letendraea helminthicola $^{\dagger}$ & CBS 884.85 & AY016362 & AY016345 & - \\
\hline Letendraea padouk $k^{\dagger}$ & CBS 485.70 & AY849951 & GU296162 & - \\
\hline Massaria platani ${ }^{\dagger}$ & AFTOL-ID 1574 & DQ678065 & & \\
\hline Massarina arundinacea ${ }^{\dagger}$ & CBS 619.86 & DQ813509 & DQ813513 & - \\
\hline Massarina cisti & CBS 266.62 & FJ795447 & FJ795490 & - \\
\hline Massarina eburnea ${ }^{\dagger}$ & CBS 473.64 & GU301840 & GU296170 & GU349040 \\
\hline Montagnula anthostomoides ${ }^{\dagger}$ & CBS 615.86 & GU205223 & GU205246 & - \\
\hline Montagnula opulenta ${ }^{\dagger}$ & CBS 168.34 & DQ678086 & AF164370 & - \\
\hline Morosphaeria ramunculicola ${ }^{\dagger}$ & BCC 18405 & GQ925854 & GQ925839 & - \\
\hline Morosphaeria ramunculicola & JK5304B & GU479794 & GU479760 & \\
\hline Morosphaeria velataspora ${ }^{\dagger}$ & BCC 17058 & GQ925851 & GQ925840 & - \\
\hline Morosphaeria velataspora $^{\dagger}$ & BCC 17059 & GQ925852 & GQ925841 & - \\
\hline Neottiosporina paspali & CBS 331.37 & EU754172 & EU754073 & GU349079 \\
\hline Ophiosphaerella sasicola ${ }^{\dagger}$ & MAFF 239644 & AB524599 & AB524458 & AB539111 \\
\hline Paraconiothyrium minitans ${ }^{\dagger}$ & CBS 122788 & EU754173 & EU754074 & GU349083 \\
\hline Paraphaeosphaeria michotii ${ }^{\dagger}$ & CBS 652.86 & GU456325 & GU456304 & GU456266 \\
\hline Paraphaeosphaeria michotii ${ }^{\dagger}$ & CBS 591.73 & GU456326 & GU456305 & GU456267 \\
\hline Stagonospora macropycnidia ${ }^{\dagger}$ & CBS 114202 & GU301873 & GU296198 & GU349026 \\
\hline Tingoldiago graminicola & KH 68 & AB521743 & AB521726 & - \\
\hline Tingoldiago graminicola ${ }^{\dagger}$ & KT 891 & AB521744 & AB521727 & - \\
\hline Tingoldiago graminicola ${ }^{\dagger}$ & KH 155 & AB521745 & AB521728 & - \\
\hline Trematosphaeria pertusa & CBS 122368 & FJ201990 & FJ201991 & GU456276 \\
\hline Trematosphaeria pertusa ${ }^{\dagger}$ & CBS 122371 & GU301876 & GU348999 & GU349085 \\
\hline
\end{tabular}

\footnotetext{
$\dagger$ ex-type sequences.

newly generated sequences.
} 


\section{Taxonomy}

Keissleriella dactylidis Singtripop, Camporesi, Hyde, sp. nov.

Index Fungorum number: IF 551020, Facesoffungi number: FoF 00567, Fig. 2.

Etymology: The species epithet refers to host genus "Dactylis".

Holotype: MFLU 14-0107.

Saprobic on a dead stem of Dactylis glomerata. Sexual morph: Ascomata 142-192 $\mu \mathrm{m}$ high $\times 138-175 \mu \mathrm{m}$ diam. $(\bar{x}=161 \times 177 \mu \mathrm{m}$, $n=5$ ), immersed in the host tissue, globose to subglobose, dark grey to black, rounded, dimidiate, erumpent through the covering layers of the host tissue, ostiole central. Ostiole 53-74 $\mu \mathrm{m}$ high $\times 55-79 \mu \mathrm{m}$ diam. $(\bar{x}=60 \times 69 \mu \mathrm{m}, n=5)$, papillate, dark brown, smooth, containing brown to dark brown setae of periphyses; 47-72 $\mu \mathrm{m}$ long. Peridium 14-48 $\mu \mathrm{m}$ wide, comprising two layers, outer layer heavily pigmented, comprising brown to dark brown cells of Textura angularis, inner layer composed of hyaline thinwalled cells of Textura angularis. Hamathecium comprising 100-119 $\mu \mathrm{m}$ high, filamentous, occasionally branched, septate, hyaline, pseudoparaphyses. Asci 58-83 $\mu \mathrm{m} \times 11-13 \mu \mathrm{m} \quad(\bar{x}=$ $68 \times 12 \mu \mathrm{m}, n=10)$, 8-spored, bitunicate, fissitunicate, broadly cylindrical, short-pedicellate, apically rounded, smooth-walled, with an ocular chamber. Ascospores 15-20 ×5-6 $\mu \mathrm{m}(\bar{x}=19 \times 5 \mu \mathrm{m}$, $n=20$ ), 2-4-overlapping seriate, broadly ellipsoid to slightly fusiform, somewhat curved, ends usually rounded, hyaline, 4-5-septate, not or only slightly constricted at the septa, surrounding by a thin gelatinous sheath. Asexual morph: undetermined.

Colonies in culture: Ascospores germinating on MEA or PDA within 36-48 h. Colonies growing on MEA or PDA, reaching $2.5 \mathrm{~cm}$ diam. after 20 days at $18-20^{\circ} \mathrm{C}$, white aerial mycelium on surface and reverse of culture whitish to pale yellow.

Material examined: ITALY, Province of Trento, Marilleva, on a dead stem of Dactylis glomerata (Poaceae), 5 August 2012, Erio Camporesi, IT620 (MFLU 14-0107, holotype); ibid., (PDD, isotype); ex-type living culture, MFLUCC 13-0751, CBS.

Keissleriella is characterized by ascomata with papilla filled with black setae, hyaline and 1-septate to multi-septate ascospores ${ }^{6,15,16}$. Nine species of Keissleriella including our new species have been described from the host family Poaceae (Table 2). Keissleriella dactylidis differs from other species of Keissleriella, based mainly on morphological charac- ters of ascospores, but is most similar to K. culmifida (P. Karst.) S.K. Bose and K. taminensis (H. Wegelin) S.K. Bose. Although K. dactylidis and K. taminensis have hyaline ascospores with 4-5 septa and are surrounded by a mucilaginous sheath ${ }^{31}$, K. dactylidis differs from $K$. taminensis in having the ellipsoid to cylindrical ascospores, longer periphyses and smaller ascomata. Keissleriella culmifida differs from $K$. dactylidis which has 3-septate, fusiform ascospores and shorter periphyses ${ }^{31}$.

Phylogenetic analysis (Fig. 1) of combined SSU, LSU, and TEF gene data showed that Keissleriella dactylidis forms a separate clade within the genus Keissleriella sister to K. linearis E. Müll. ex Dennis with relatively high bootstrap support (65\%). Keissleriella linearis differs from $K$. dactylidis in having 1-3-septate ascospores lacking a mucilaginous sheath and larger ascomata ${ }^{32}$.

Lentithecium lineare (三 Keissleriella linearis E. Müll., Dennis) was introduced by Zhang et al $^{12}$ as a new combination in Lentithecium as it grouped with Lentithecium sp. However, Zhang et al ${ }^{12}$ had not included any Keissleriella strains in their study. In our study Lentithecium lineare groups with Keissleriella species (Fig. 1) and is treated as Keissleriella linearis (Dennis, 1964) (Fig. 3).

Keissleriella linearis E. Müll. ex Dennis, Kew Bull. 19(1): 120 (1964).

$\equiv$ Lentithecium lineare (E. Müll. ex Dennis) Hyde et al, Fungal Diversity 38: 236 (2009).

Saprobic on dead stems of Phragmites australis. Sexual morph: Ascomata 598-752 $\mu \mathrm{m}$ high $\times 371-491 \mu \mathrm{m}$ diam. $(\bar{x}=650 \times 438 \mu \mathrm{m}$, $n=5$ ), immersed or erumpent on the host tissue, globose to subglobose, dark grey to black, rounded, dimidiate, erumpent through the covering layers of the host tissue, ostiole centre. Ostiole 91-143 $\mu \mathrm{m}$ high $\times 73-117 \mu \mathrm{m}$ diam. $(\bar{x}=118 \times 98 \mu \mathrm{m}, n=4)$, papillate, pale brown to brown, smooth, containing brown to dark brown setae of periphyses; 39-52 $\mu \mathrm{m}$ long. Peridium 35-94 $\mu \mathrm{m}$ wide, comprising brown to dark brown cells of Textura angularis. Hamathecium comprising 122-134 $\mu \mathrm{m}$ high, filamentous, occasionally branched, septate, hyaline, pseudoparaphyses. Asci 117-129×16-19 $\mu \mathrm{m}(\bar{x}=123 \times$ $17 \mu \mathrm{m}, n=5$ ), 8-spored, bitunicate, broadly cylindrical, smooth-walled, with an ocular chamber. Ascospores $36-39 \times 9-10 \mu \mathrm{m}(\bar{x}=37 \times 9 \mu \mathrm{m}, n=10)$, 2-4 overlapping seriate, broadly ellipsoid to slightly fusiform, somewhat curved, ends usually rounded, hyaline, 1-3-septate, not or only slightly constricted at the septa, without surrounding by a thin gelatinous sheath. Asexual morph: undetermined. 

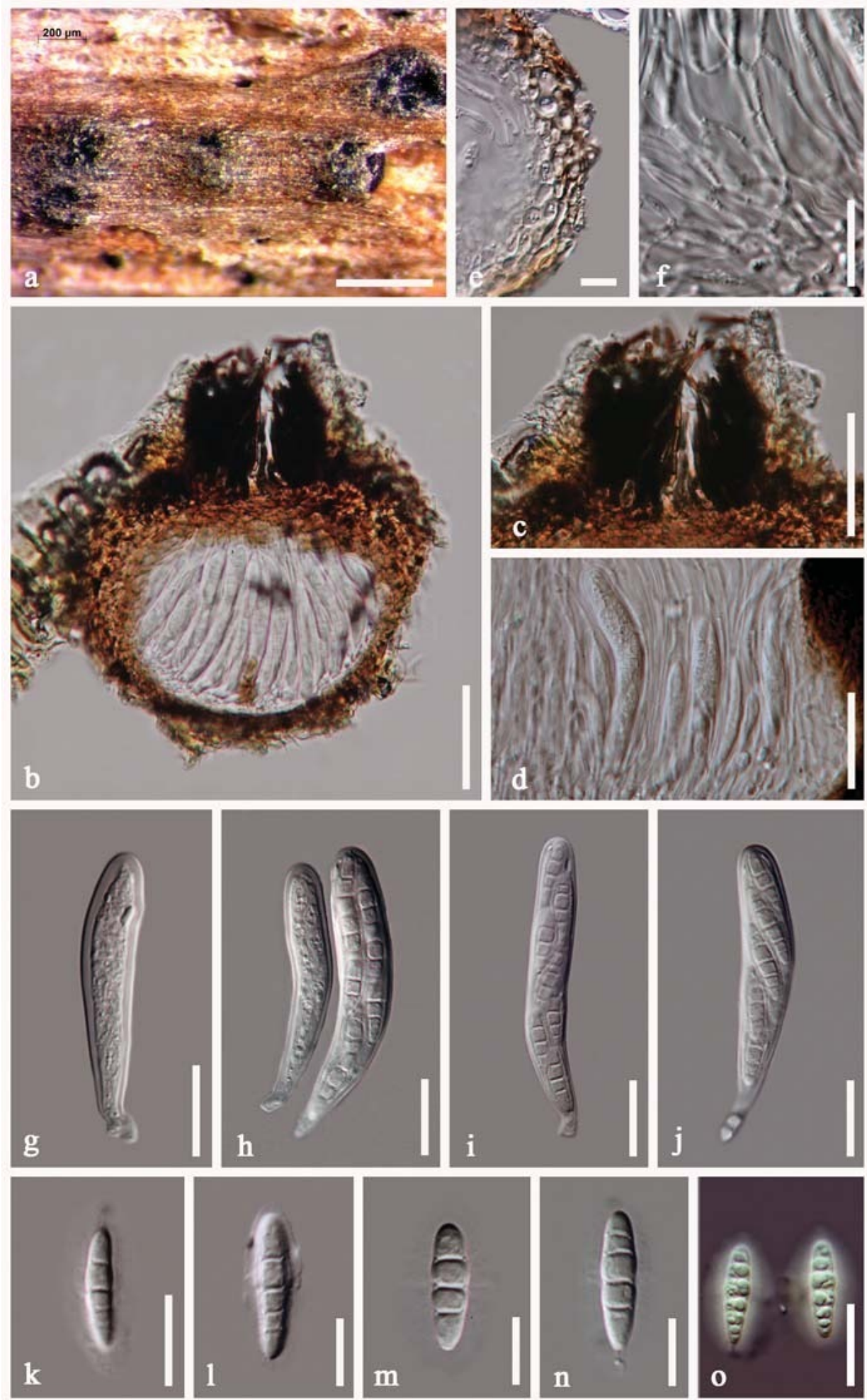

Fig. 2 Keissleriella dactylidis: (a) appearance of ascomata on host; (b) vertical section through ascoma; (c) periphyses in ostiole; (d) asci with pseudoparaphyses; (e) section of peridium; (f) pseudoparaphyses; ( $\mathrm{g}-\mathrm{j}$ ) immature and mature asci; (k-n) mature ascospores; (o) ascospore in Indian ink. Scale bars: $a=400 \mu \mathrm{m}$; b-d, f = $50 \mu \mathrm{m}$; e, $\mathrm{k}-\mathrm{n}=10 \mu \mathrm{m}$; $\mathrm{g}-\mathrm{j}, \mathrm{o}=20 \mu \mathrm{m}$. 

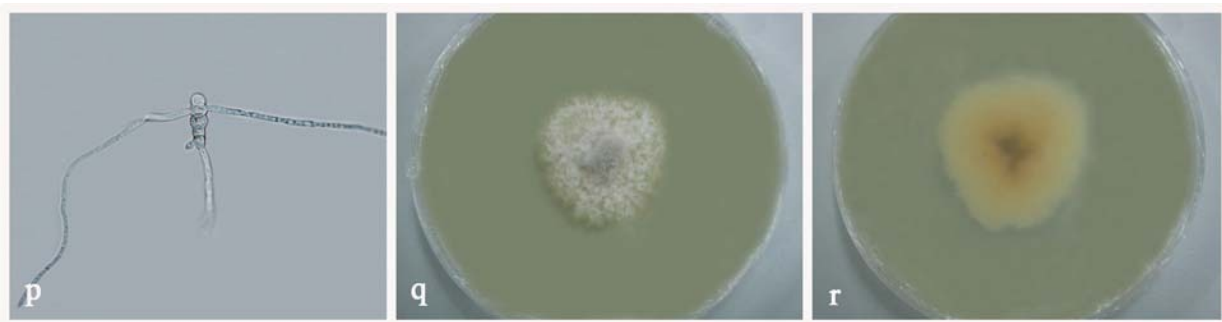

Fig. 2 (Cont.) Keissleriella dactylidis (from culture of holotype): (p) germinated ascospore; (q) character of colony on MEA from above; (r) character of colony on PDA medium from below.
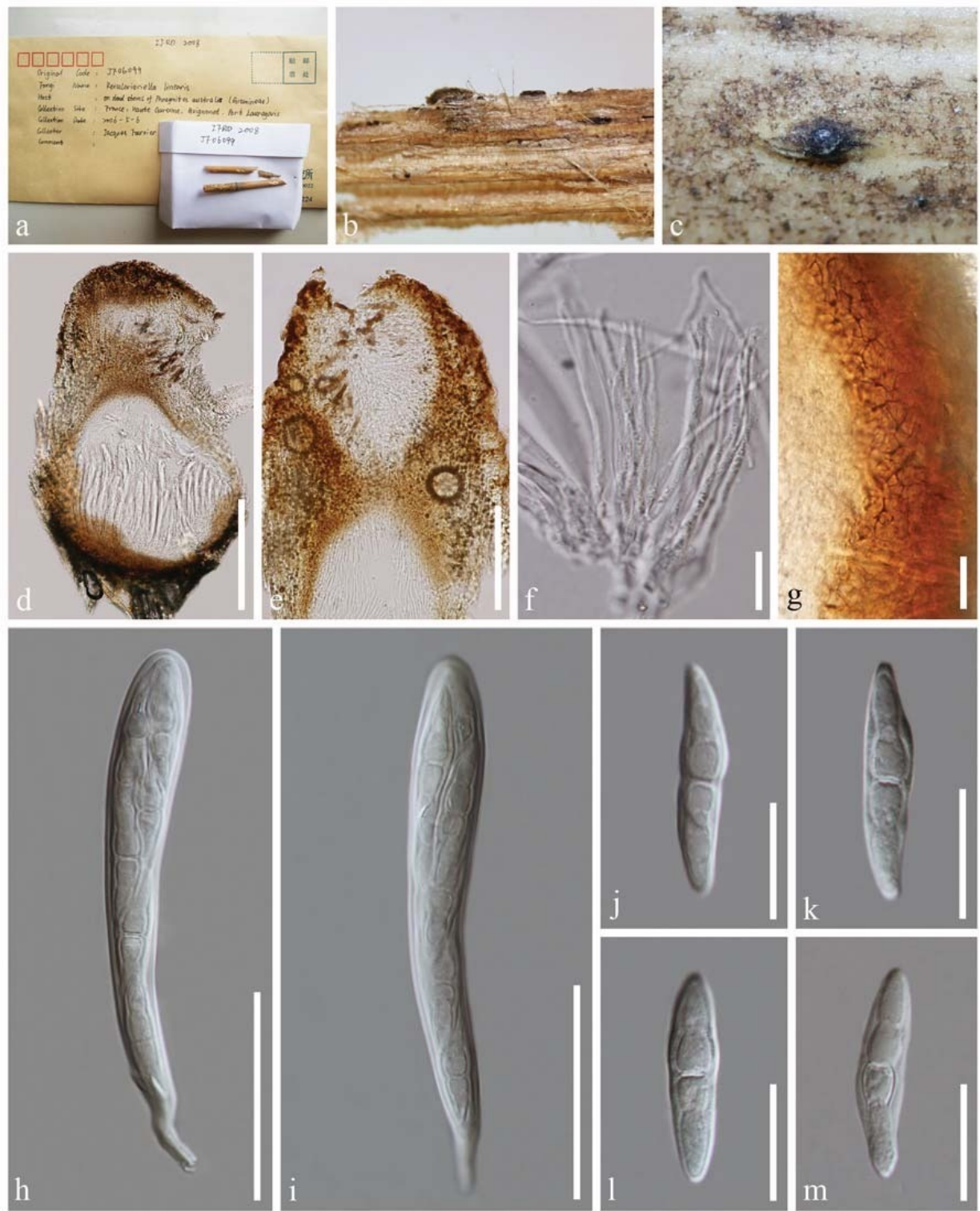

Fig. 3 Keissleriella linearis: (a) material label; (b,c) appearance of ascomata on host; (d) vertical section through ascoma; (e) periphyses in ostiole; (f) pseudoparaphyses; ( $g$ ) section of peridium; (h,i) mature asci; (j-m) ascospore. Scale bars: $d=200 \mu \mathrm{m}$; $e=100 \mu \mathrm{m} ; \mathrm{h}, \mathrm{i}=50 \mu \mathrm{m} ; \mathrm{f}, \mathrm{g}, \mathrm{j}-\mathrm{m}=20 \mu \mathrm{m}$. 
Material examined: FRANCE, Haute Garonne, Avignonet, Port Laurgaris, on a dead stem of Phragmites australis (Gramineae), 6 May 2006, Jacques Fournier, IFRD 2008.

Notes: We examined this type of Keissleriella linearis which has characters typical of the genus (Fig. 3). The setae were not illustrated in Zhang et $\mathrm{al}^{12}$.

Acknowledgements: The authors extend their sincere appreciations to the Deanship of Scientific Research at King Saud University for its funding this prolific research group (PRG-1436-09). We would like to thank for Mae Fah Luang University grant "Taxonomy and phylogeny of selected families of Dothideomycetes (Grant number: 56101020032)" for supporting this study. Thank for Humidtropics, a CGIAR Research Programme that aims to develop new opportunities for improved livelihoods in a sustainable environment, for partially funding this work, Chinese Academy of Sciences, project number 2013T2S0030, for the award of Visiting Professorship for Senior International Scientists at Kunming Institute of Botany, Plant Germplasm and Genomics Centre in Germplasm Bank of Wild Species, Kunming Institute of Botany for the help of molecular work. Chonticha Singtripop thanks to Rungtiwa Phookamsak and Dr Sajeewa S.N. Maharachchikumbura for their valuable suggestions and help.

Table 2 Synopsis of the characteristics among Keissleriella dactylidis and other species of genus Keissleriella reported from Poaceae.

\begin{tabular}{|c|c|c|c|c|c|}
\hline Fungus name & Host & Ascomata & $\begin{array}{l}\text { Setae in papilla } \\
\text { (periphyses) }\end{array}$ & Ascospore & Ref. \\
\hline K. dactylidis & Dactylis glomerata & $\begin{array}{l}142-192 \times 138-175 \mu \mathrm{m}, \\
\text { globose to subglobose, } \\
\text { immersed }\end{array}$ & $\begin{array}{l}\text { 47-72 } \mu \mathrm{m} \text { long, } \\
\text { dark brown }\end{array}$ & $\begin{array}{l}15-20 \times 5-6 \mu \mathrm{m} \text {, } \\
\text { ellipsoid to cylindrical, } \\
\text { hyaline, } 4-5 \text {-septate, } \\
\text { surrounded by } \\
\text { a mucilaginous sheath }\end{array}$ & $\begin{array}{l}\text { This } \\
\text { study }\end{array}$ \\
\hline $\begin{array}{l}\text { K. caudata } \\
\text { (E. Müll.) } \\
\text { Corbaz }\end{array}$ & Triticum sp. & $\begin{array}{l}\text { 80-230 } \mu \mathrm{m} \text { diameter, } \\
\text { flask-shaped, } \\
\text { immersed to erumpent }\end{array}$ & $\begin{array}{l}24-40 \mu \mathrm{m} \text { long, } \\
\text { light brown }\end{array}$ & $\begin{array}{l}10.5-13 \times 4-4.5 \mu \mathrm{m} \text {, } \\
\text { fusiform to elliptical, } \\
\text { hyaline, } 1 \text { septate }\end{array}$ & 30 \\
\hline $\begin{array}{l}\text { K. culmifida } \\
\text { (P. Karst.) } \\
\text { S.K. Bose }\end{array}$ & $\begin{array}{l}\text { Agrostis sp., } \\
\text { Calamagrostis } \\
\text { arundinacea, } \\
\text { Leymus arenarius, } \\
\text { Nardus stricta, } \\
\text { Phleum pratense, } \\
\text { Triticum spp., } \\
\text { Poa compressa, } \\
\text { Poa normalis, } \\
\text { Poa pratensis }\end{array}$ & $\begin{array}{l}140-250 \times 115-200 \mu \mathrm{m} \text {, } \\
\text { globose or pear-shaped, } \\
\text { immersed }\end{array}$ & $\begin{array}{l}20-40 \times 3-4 \mu \mathrm{m}, \\
\text { dark brown }\end{array}$ & $\begin{array}{l}10-25 \times 4.5-6 \mu \mathrm{m}, \\
\text { fusiform, hyaline, } \\
3 \text {-septate, } \\
\text { surrounded by } \\
\text { a mucilaginous sheath }\end{array}$ & 31 \\
\hline $\begin{array}{l}\text { K. gloeospora } \\
\text { (Berk., Curr.) } \\
\text { S.K. Bose }\end{array}$ & Unidentified grass & $\begin{array}{l}200-300 \mu \mathrm{m} \text { diameter, } \\
\text { globose or pear-shaped, } \\
\text { immersed }\end{array}$ & $\begin{array}{l}\text { 60-90 } \mu \mathrm{m} \text { long, } \\
\text { dark brown }\end{array}$ & $\begin{array}{l}23-33 \times 6-8 \mu \mathrm{m} \text {, } \\
\text { fusiform to ellipsoid, } \\
\text { hyaline, } 4-6 \text {-septate }\end{array}$ & 31 \\
\hline $\begin{array}{l}\text { K. linearis } \\
\text { E. Müll. } \\
\text { ex Dennis }\end{array}$ & $\begin{array}{l}\text { Phragmites } \\
\text { australis }\end{array}$ & $\begin{array}{l}584-762 \times 369-495 \mu \mathrm{m}, \\
\text { globose to subglobose, } \\
\text { immersed or erumpent }\end{array}$ & $\begin{array}{l}34-48 \mu \mathrm{m} \text { long, } \\
\text { brown to dark brown }\end{array}$ & $\begin{array}{l}32-42 \times 8-10 \mu \mathrm{m}, \\
\text { ellipsoid to fusiform, } \\
\text { hyaline, } 1-3 \text {-septate }\end{array}$ & 32 \\
\hline $\begin{array}{l}\text { K. poagena } \\
\text { Crous, } \\
\text { Quaedvlieg }\end{array}$ & Poa sp. & $\begin{array}{l}250 \mu \mathrm{m} \text { diameter, } \\
\text { erumpent to superficial }\end{array}$ & $\begin{array}{l}60 \mu \mathrm{m} \text { long, } \\
\text { dark brown }\end{array}$ & $\begin{array}{l}20-25 \times 4-5 \mu \mathrm{m} \text {, } \\
\text { fusiform to ellipsoid, } \\
\text { hyaline, } 3 \text {-septate, } \\
\text { surrounded by } \\
\text { a mucilaginous sheath }\end{array}$ & 33 \\
\hline $\begin{array}{l}\text { K. taminensis } \\
\text { (H. Wegelin) } \\
\text { S.K. Bose }\end{array}$ & $\begin{array}{l}\text { Agropyron repens, } \\
\text { Bromus inermis, } \\
\text { Festuca spp., } \\
\text { Poa nemoralis }\end{array}$ & $\begin{array}{l}180-300 \times 150-200 \mu \mathrm{m} \text {, } \\
\text { globose or pear-shaped, } \\
\text { immersed }\end{array}$ & $\begin{array}{l}20-30 \times 3.5-5.5 \mu \mathrm{m} \text {, } \\
\text { dark brown }\end{array}$ & $\begin{array}{l}18-23 \times 4.5-5 \mu \mathrm{m}, \\
\text { fusiform, hyaline, } \\
4-5 \text {-septate, surrounded } \\
\text { by a mucilaginous sheath }\end{array}$ & 31 \\
\hline
\end{tabular}




\section{REFERENCES}

1. Hyde KD, Jones EBG, Liu JK, Ariyawansa H, Boehm E, Boonmee S, Braun U, Chomnunti P, et al (2013) Families of Dothideomycetes. Fungal Divers 63, 1-313.

2. Zhang Y, Crous PW, Schoch CL, Hyde KD (2012) Pleosporales. Fungal Divers 53, 1-221.

3. Schoch CL, Crous PW, Groenewald JZ, Boehm EWA, Burgess TI, de Gruyter J, de Hoog GS, Dixon LJ, et al (2009) A class-wide phylogenetic assessment of Dothideomycetes. Stud Mycol 64, 1-15.

4. Kirk PM, Cannon PF, Minter DW, Stalpers JA (2008) Ainsworth \& Bisby's Dictionary of the Fungi, 10th edn, CABI, Wallingford, UK.

5. Manamgoda DS, Cai L, McKenzie EHC, Crous PW, Madrid H, Chukeatirote E, Shivas RG, Tan YP, Hyde KD (2012) A phylogenetic and taxonomic reevaluation of the Bipolaris - Cochliobolus - Curvularia Complex. Fungal Divers 56, 131-44.

6. Hyde KD, Nilsson RH, Alias SA, Ariyawansa HA, Blair JE, Cai L, de Cock AWAM, Dissanayake AJ, et al (2014) One stop shop: backbones trees for important phytopathogenic genera: I. Fungal Divers 67, 21-125.

7. Ariyawansa HA, Tanaka K, Thambugala KM, Phookamsak R, Tian Q, Camporesi E, Hongsanan S, Monkai J, et al (2014) A molecular phylogenetic reappraisal of the Didymosphariaceae (= Montagnulaceae). Fungal Divers 68, 69-104.

8. Wijayawardene NN, Crous PW, Kirk PM, Hawksworth DL, Boonmee S, Braun U, Dai DQ, D’Souza MJ, et al (2014) Naming and outline of Dothideomycetes-2014 including proposals for the protection or suppression of generic names. Fungal Divers 69, 1-55.

9. Zhang Y, Fournier J, Crous PW, Pointing SB, Hyde KD (2009) Phylogenetic and morphological assessment of two new species of Amniculicola and their allies (Pleosporales). Persoonia 23, 48-54.

10. Ariyawansa HA, Jones EBG, Suetrong S, Alias SA, Kang JC, Hyde KD (2013) Halojulellaceae a new family of the order Pleosporales. Phytotaxa 130, 14-24.

11. Liu YX, Hyde KD, Ariyawansa HA, Li WJ, Zhou DQ, Yang YL, Chen YM, Liu ZY (2013) Shiraiaceae, new family of Pleosporales (Dothideomycetes, Ascomycota). Phytotaxa 103, 51-60.

12. Zhang Y, Wang HK, Fournier J, Crous PW, Jeewon R, Pointing SB, Hyde KD (2009) Towards a phylogenetic clarification of Lophiostoma / Massarina and morphologically similar genera in the Pleosporales. Fungal Divers 38, 225-51.

13. Zhang Y, Schoch CL, Fournier J, Crous PW, De Gruyter J, Woudenberg JHC, Hirayama K, Tanaka K, Pointing SB, Hyde KD (2009) Multi-locus phylogeny of Pleosporales: a taxonomic, ecological and evolutionary re-evaluation. Stud Mycol 64, 85-102.

14. von Höhnel FXR (1919) Fragmente zur Mykologie
(XXIII. Mitteilung, Nr. 1154 bis 1188). Sitzungsber Akad Wiss Wien Math Naturwiss Kl Abt 1 128, 535-625.

15. Barr ME (1990) Melanommatales (Loculoascomycetes), North American Flora, Series II, Part 13, New York Botanical Garden Press, Bronx, NY.

16. Quaedvlieg W, Verkley GJM, Shin H-D, Barreto RW, Alfenas AC, Swart WJ, Groenewald JZ, Crous PW (2013) Sizing up Septoria. Stud Mycol 75, 307-90.

17. Liu JK, Hyde KD, Jones EBG, Ariyawansa HA, Bhat DJ, Boonmee S, Maharachchikumbura S, McKenzie EHC, et al (2015) Fungal Diversity Notes 1-110: Taxonomic and phylogenetic contributions to fungal species. Fungal Divers 72, 1-197.

18. Munk A (1957) Danish Pyrenomycetes. Dan Bot Ark 17, 1-491.

19. von Arx JA, Müller E (1975) A re-evaluation of the bitunicate Ascomycetes with keys to families and genera. Stud Mycol 9, 1-159.

20. Lumbsch HT, Huhndorf SM (2007) Outline of Ascomycota. Myconet 13, 1-58.

21. Chomnunti P, Hongsanan S, Aguirre-Hudson B, Tian Q, Peršoh D, Dhami MK, Alias AS, Xu J, et al (2014) The sooty moulds. Fungal Divers 66, 1-36.

22. White TJ, Bruns TD, Lee S, Taylor J (1990) Amplification and direct sequencing of fungal ribosomal RNA genes for phylogenetics. In: Innis MA, Gelfand DH, Sninsky JJ, White TJ (eds) PCR Protocols: a Guide to Methods and Applications, Academic, New York, pp 315-22.

23. Vilgalys R, Hester M (1990) Rapid genetic identification and mapping of enzymatically amplified ribosomal DNA from several Cryptococcus species. $J$ Bacteriol 172, 4238-46.

24. Rehner S (2001) Primers for Elongation Factor 1- $\alpha$ (EF1- $\alpha)$, available at www.aftol.org/pdfs/ EF1primer.pdf.

25. Hall TA (1999) BioEdit: a user-friendly biological sequence alignment editor and analysis program for Windows 95/98/NT. Nucleic Acids Symp Ser 41, 95-8.

26. Thompson JD, Gibson TJ, Plewniak F, Jeanmougin F, Higgins DG (1997) The CLUSTAL_X Windows interface: flexible strategies for multiple sequence alignment aided by quality analysis tools. Nucleic Acids Res 25, 4876-82.

27. Swofford DL (2002) PAUP: Phylogenetic Analysis Using Parsimony Version 4.0b10, Sinauer Associates, Sunderland, MA.

28. Kishino H, Hasegawa M (1989) Evaluation of the maximum likelihood estimate of the evolutionary tree topologies from DNA sequence data, and the branching order in Hominoidea. J Mol Evol 29, 170-9.

29. Page RDM (1996) TreeView: an application to display phylogenetic trees on personal computers. Comput Appl Biosci 12, 357-8. 
30. Corbaz R (1956) Recherches sur le genre Didymella Sacc. Phytopathol Z 28, 375-414.

31. Bose SK (1961) Studies on Massarina Sacc. and related genera. $J$ Phytopathol 41, 151-213.

32. Dennis RWG (1964) The fungi of the Isle of Rhum. Kew Bull 19, 77-127.

33. Crous PW, Shivas RG, Quaedvlieg W, van der Bank M, Zhang Y, Summerell BA, Guarro J, Wingfield MJ, et al (2014) Fungal Planet description sheets: 214 280. Persoonia 32, 184-306. 